\title{
Depression, diabetes, and dementia: Is there a role for insulin?
}

\author{
Danielle S. Cha
}

\section{University of Toronto}

Cognitive deficits are increasingly being recognized as a consistent, non-specific, and clinically significant feature across neuropsychiatric and metabolically-based medical conditions..$^{1-3}$ Moreover, converging evidence suggests that individuals with comorbid neuropsychiatric (i.e., major depressive disorder and Alzheimer's disease) and/ or metabolically-based medical conditions (i.e., type II diabetes mellitus and major depressive disorder) are at elevated risk for hastening the progression of cognitive decline and neurodegeneration. ${ }^{2,3}$ The phenotypic and neurobiological overlap between frequently co-occurring neuropsychiatric disorders and metabolically-based medical conditions suggests the possibility for shared pathophysiological mechanisms. ${ }^{4}$ However, relatively few reports have aimed to describe potential mechanisms underlying the relationship between major depressive disorder (MDD), type II diabetes mellitus (T2DM), and Alzheimer's disease (AD).

$M D D, T 2 D M$, and $A D$ are among the most common disorders worldwide and represent major public health concerns. ${ }^{5}$ It has been amply documented that MDD represents both a risk factor and a prodrome for AD. ${ }^{6}$ Moreover, cognitive impairments are reported to be a common and persisting abnormality among individuals with MDD. ${ }^{6}$ For example, it was reported that individuals with MDD experience decreases in memory by approximately 2-3\% following each major depressive episode. ${ }^{1}$ Furthermore, evidence suggests that an estimated $10-15 \%$ of AD cases are attributable to depression and a $25 \%$ reduction in depression prevalence would result in approximately 830,000 fewer AD cases worldwide. ${ }^{6}$ Conversely, approximately $20-30 \%$ of patients with AD have been reported to suffer from MDD. ${ }^{6}$ In keeping with this view, individuals with T2DM are reported to be $52 \%$ more likely to develop MDD when compared to the general population. ${ }^{5}$ Moreover, T2DM has been reported to significantly and independently increase risk for $A D$ by
$39 \%$ when compared to the general population. ${ }^{5}$ These foregoing observations indicate that the cognitive changes that occur in a subpopulation of individuals with MDD and T2DM are progressive, suggesting the potential for shared pathophysiological substrates between MDD, T2DM, and AD.

Disease modeling in neuropsychiatric disorders and metabolically-based medical conditions indicate that abnormal central insulin signaling is implicated in alterations of neuronal integrity and function, affecting synaptic signaling and discrete neural circuits. ${ }^{4}$ Replicated evidence indicates that cross-talk between central and peripheral insulin is critical to elucidating the relationship between metabolic disturbances (e.g., insulin resistance) and neuropsychiatric disorders (e.g., MDD and AD). ${ }^{4}$ Taken together, the role of insulin in normal and abnormal peripheral and central nervous system (CNS) functioning provides a framework for characterizing its contribution to the pathoetiology, progression, and potential treatment of MDD, T2DM, and AD.

Recent interest in insulin signaling and its relationship with disorders affecting cognition is associated with its critical role as a neuropeptide, exerting pleiotropic effects involved in neurotrophism, neuroplasticity, and neuromodulation. ${ }^{4,7}$ Insulin derived from the pancreas is transported via a saturable, receptor-mediated process across the blood brain barrier (BBB) to prompt the insulin-mediated signaling pathways involved in brain bioenergetics, reward circuits, as well as the regulation of normal emotional and cognitive brain functions. ${ }^{4,7}$ Furthermore, insulin signaling in the CNS has been reported to prevent the pathological binding of amyloid beta $(A \beta)$ oligomers by up-regulating the expression of insulin degrading enzyme (IDE), which also degrades $A \beta .3,4,7$

Chronic insulin-resistance related hyperinsulinemia results in the down-regulation of insulin receptor expression - 
C along the BBB impeding the transport of insulin into the brain, and chronically reducing IDE levels. ${ }^{3,4,7}$ For example, peripheral insulin resistance has been associated with both T2DM and MDD. ${ }^{3,48}$ Moreover, emerging evidence suggests that $A \beta$ peptides may play a distinct role in depression etiology in addition to its interaction with $A D$ pathology. ${ }^{9}$ Taken together, the relationship between insulin, its relevant signaling pathways, and $A \beta$ may represent a potential mechanism through which impairments in affect, cognition, and metabolic health may synergistically perpetuate neurodegeneration via separate but related systems, further elucidating the pathoetiological link and progressive relationship between MDD, T2DM, and AD.

Available evidence provides the impetus to further refine potential mechanisms and molecular pathways subserving these disorders in order to more effectively treat these populations by aiming to reduce and prevent cognitive impairments and decline, and downstream neurodegeneration. For instance, the administration of intranasal insulin has been reported to improve memory and measures of mood (e.g., depression, self-confidence, overall feeling of well-being) in healthy individuals as well as individuals with $A D .^{10}$ Notwithstanding accumulating evidence for the relevance of cognitive dysfunction in MDD and T2DM, robust therapeutic interventions specifically targeting cognitive dysfunction across neuropsychiatric disorders and/or metabolically-based medical conditions are not currently available, largely due to a paucity of empirical evidence. Thus, investigations that aim to examine the contribution of various effector systems and molecular pathways that converge across these clinical populations may provide the basis for novel treatment avenues specific to ameliorating and preventing cognitive dysfunction and thereby AD.

\section{References}

1. Mclntyre RS, Cha DS, Soczynska JK, Woldeyohannes HO, Gallaugher LA, Kudlow P, et al. Cognitive Deficits And Functional Outcomes In Major Depressive Disorder: Determinants, Substrates, And Treatment Interventions. Depress Anxiety 2013 Mar 6.

2. Rapp MA, Schnaider-Beeri M, Wysocki M, Guerrero-Berroa E, Grossman HT, Heinz $A$, et al. Cognitive decline in patients with dementia as a function of depression. Am J Geriatr Psychiatry 2011 Apr;19(4):357-63.

3. Umegaki $H$, Hayashi $T$, Nomura $H$, Yanagawa $M$, Nonogaki $Z$, Nakshima $\mathrm{H}$, et al. Cognitive dysfunction: an emerging concept of a new diabetic complication in the elderly. Geriatr Gerontol Int 2013 Jan;13(1):28-34.

4. Kaidanovich-Beilin O, Cha DS, McIntyre RS. Crosstalk between metabolic and neuropsychiatric disorders. F1000 Biol Rep 2012;4:14.

5. Katon W, Lyles CR, Parker MM, Karter AJ, Huang ES, Whitmer RA. Association of depression with increased risk of dementia in patients with type 2 diabetes: the Diabetes and Aging Study. Arch Gen Psychiatry 2012 Apr;69(4):410-7.

6. Enache D, Winblad B, Aarsland D. Depression in dementia: epidemiology, mechanisms, and treatment. Curr Opin Psychiatry 2011 Nov;24(6):461-72.

7. Zhao L, Teter B, Morihara T, Lim GP, Ambegaokar SS, Ubeda OJ, et al. Insulindegrading enzyme as a downstream target of insulin receptor signaling cascade: implications for Alzheimer's disease intervention. J Neurosci. 2004 Dec 8;24(49):11120-6.

8. Kan C, Silva N, Golden SH, Rajala U, Timonen M, Stahl D, et al. A systematic review and meta-analysis of the association between depression and insulin resistance. Diabetes Care 2013 Feb;36(2):480-9.

9. Direk N, Schrijvers EM, de Bruijn RF, Mirza S, Hofman A, Ikram MA, et al. Plasma amyloid beta, depression, and dementia in community-dwelling elderly. J Psychiatr Res 2013 Apr;47(4):479-85.

10. Benedict C, Hallschmid M, Schultes B, Born J, Kern W. Intranasal insulin to improve memory function in humans. Neuroendocrinology 2007;86(2):136-42.

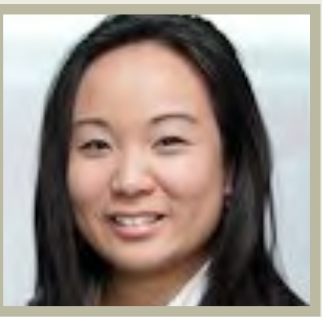

\section{Danielle S. Cha}

Danielle S. Cha completed her undergraduate studies at the University of Toronto and is currently completing her Master's in Medical Science under the supervision of Dr. Roger S. McIntyre at the Mood Disorders Psychopharmacology Unit, University Health Network under the aegis of the Institute of Medical Science, University of Toronto. She has a particular interest in the interface between mood disorders and medical comorbidity with an emphasis on their functional implications. 